\title{
VARIATIONS DES REPONSES DE L'OVAIRE DE LA BREBIS A DES DOSES CROISSANTES D'HORMONES GONADOTROPES D'ORIGINES DIVERSES
}

PAR

\section{DAUZIER, C. THIBA ULT et Suzanne WINTENBERGER}

Station de Physiologie Animale, Centre national de Recherches zootechniques, Jouy-en-Josas (Seine-et-Oise) $\left.{ }^{(}{ }^{1}\right)$

Chez les mammifères domestiques à faible fécondité, la superovulation artificielle a été recherchée, soit pour améliorer rapidement la productivité d'un troupeau (ZAvadowșki), soit pour disposer d'un nombre d'œufs appréciable, permettant d'étudier des problèmes intéressant indirectement la production animale, tels que la fécondation in vitrn, la transplantation des œufs d'une femelle donatrice dans une femelle réceptrice, la polyploïdie.

Selon le but que l'on recherche, l'importance numérique de la superovulation varie considérablement : pour l'élevage ovin par exemple, on doit tendre vers un maximum de 3 ovulations, donc différencier un ou deux follicules supplémentaires. Au contraire, si l'animal est considéré comme un donneur d'œufs, il est souhaitable d'obtenir des pontes groupées aussi nombreuses que possible.

Or en I948, Thibault, Ortavant et Laplaud, en utilisant du sérum de jument gravide rapportèrent des résultats d'une telle homogénéité que l'on pouvait penser qu'une méthode sûre était obtenue pour différencier régulièrement chez toutes les femelles traitées un ou deux follicules supplémentaires permettant ainsi l'application de cette technique à l'élevage ovin. Mais au cours d'essais répétés de I949 à I95I, nous n'avons pas réussi à retrouver la constance des réponses enregistrées précédemment : avec des doses relativement faibles d'hormone gonadotrope, nous avons obtenu au sein d'une même race des réponses individuelles très différentes, ce qui confirme les résultats de Robinson (I950) et de ZAVADOWSKI (I95I).

Cependant, en poursuivant ces recherches, nous nous sommes aperçus qu'avec des doses pluss élevées entraînant la ponte de 6 à II œufs, on retrouvait, en toutes circonstances, une bonne homogénéité des réponses.

Afin d'arriver à une meilleure compréhension du mode d'action de

(1) Ces recherches ont été poursuivies en partie grâce aux crédits reçus du Fonds national du progrès agricole, en provenance du Fonds d'encouragement à l'industrie textile, sur l'initiative de la Fédération nationale ovine. 
1'hormone gonadotrope injectée, nous avons donc été conduits à analyser systématiquement la réponse de l'ovaire de brebis à des doses croissantes d'hormone gonadotrope d'origines diverses : hormone gonadotrope totale extraite de l'hypophyse du cheval (technique de HALEY (I950), sérum de jument gravide brut, hormone commerciale extraite du sérum de jument gravide ${ }^{(1)}$.

Les hormones ont été testées en soumettant des rates de 2 I jours à des injections répétées 6 fois toutes les $\mathrm{I} 2$ heures. Une unité Rat (U. R.) d'extrait hypophysaire correspondait à la dose permettant un accroissement de 50 à roo $\%$ du poids moyen des ovaires. Le dosage en unités internationales (U. I.) du S. J. G. et de l'hormone commerciale a été déduit de l'augmentation de poids des ovaires de rates traitées en se référant à une courbe standard.

Nous avons utilisé dans ces expériences : 95 brebis berrichonnes ou Boukhara. Élles reçurent toutes, en outre, en début de rut, une injection d'hormone gonadotrope par voie intraveineuse afin de synchroniser les ovulations, ce qui facilite grandement la recherche des oufs et l'appréciation des corps jaunes au moment de l'abatage, sans modifier le taux d'ovulation (Ortavant, Thibault, Wintenberger, I949).

\section{Résultats}

Les résultats sont entièrement groupés dans les tableaux suivants. Nous avons fait figurer le nombre de corps jaunes vrais, reconnus après examen morphologique approfondi et non le nombre d'œufs, car d'une part certains corps jaunes ne correspondent manifestement pas à 1'évolution de follicules éclatés et d'autre part des œufs en quantité non négligeable sont perdus, principalement dans le cas d'ovulations fort importantes.

Il ressort du tableau I et des graphiques I, 2 et 3 que pour des doses faibles, on observe peu de différence dans l'activité des diverses hormones, tandis qu'au fur et à mesure que les doses injectées sont plus élevées, l'extrait hypophysaire de cheval devient beaucoup plus actif sur l'ovaire de brebis. En effet si avec 40 U. R. d'extrait hypophysaire, 600 U. I. de S. J. G. et 800 U. I. d'hormone commerciale on obtient des réponses voisines de $I, 6$ et I,7 œuf par animal, il faut seulement $80 \mathrm{U}$. R. avec 1'hormone hypophysaire, soit deux fois plus pour obtenir 8 à 9 ovocytes, alors que 4800 U. I. de S. J. G. ( 8 fois plus) et 2400 U. T. d'hormone commerciale ( 3 fois plus) sont nécessaires pour obtenir la même réponse. L,e coefficient de corrélation entre le taux d'ovulation et la dose d'hormone injectée est de 0,8 avec l'extrait hypophysaire, alors qu' 1 n'est que de 0,7 avec 1 'hormone commerciale et le sérum de jument gravide.

(1) Nous remercions les Établissements Roussel qui nous ont fourni gracieusement une partie de cette hormone. 
TABLEAU I

\begin{tabular}{|c|c|c|c|c|c|}
\hline $\begin{array}{l}\text { Origine } \\
\text { de l'hormone }\end{array}$ & $\begin{array}{l}\text { Dose injectée } \\
\text { en sous-cutanée }\end{array}$ & $\begin{array}{l}\text { Nombre } \\
\text { de brebis } \\
\text { traitées }\end{array}$ & $\begin{array}{c}\text { Nombre } \\
\text { d'ovulations }\end{array}$ & $\begin{array}{c}\text { Taux } \\
\text { moyen } \\
\text { d'ovulation }\end{array}$ & $\begin{array}{l}\text { Variations } \\
\text { du taux } \\
\text { d'ovulation }\end{array}$ \\
\hline $\begin{array}{r}\text { Extrait hypophysaire } \\
\text { de cheval } \ldots \ldots \ldots\end{array}$ & 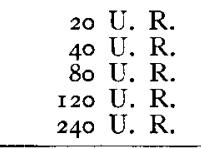 & $\begin{array}{l}2 \\
5 \\
3 \\
4 \\
4\end{array}$ & $\begin{array}{l}I-I \\
3-2-I-I-I \\
\text { IO-8-8 } \\
16-I 4-10-6 \\
30-2 I-I 7-7 \\
\end{array}$ & $\begin{array}{l}\mathrm{I} \\
\mathrm{I}, 6 \\
8,7 \\
\mathrm{I} \mathrm{I}, 5 \\
\mathrm{I} 8,7\end{array}$ & $\begin{array}{l}I \\
I-3 \\
8-10 \\
6-16 \\
7-30\end{array}$ \\
\hline Hormone commerciale. & $\begin{array}{l}800 \text { U. I. } \\
\text { I } 600 \text { U. I. } \\
2400 \text { U. I. } \\
3 \text { 200 U. I. } \\
4800 \text { U. I. }\end{array}$ & $\begin{array}{r}7 \\
\text { I } 2 \\
\\
5 \\
4 \\
4\end{array}$ & $\mid \begin{array}{l}3-2-2-I-I-I-I \\
6-6-5-3-3-2-2-2-2 \\
1-I-I \\
9-9-8-8-6 \\
9-8-6-3 \\
16-I 4-6-4\end{array}$ & $\begin{array}{l}1,6 \\
2,8 \\
8 \\
6,5 \\
10\end{array}$ & $\begin{array}{l}I-3 \\
I-6 \\
6-9 \\
3-9 \\
4-16\end{array}$ \\
\hline $\begin{array}{l}\text { Sérum de jument gra- } \\
\text { vide } \ldots \ldots \ldots \ldots \ldots \cdots\end{array}$ & $\begin{array}{l}400 \text { U. I. } \\
600 \text { U. I. } \\
800 \text { U. I. } \\
\\
\text { I } 200 \text { U. I. } \\
2400 \text { U. I. } \\
4800 \text { U. I. }\end{array}$ & $\begin{array}{r}4 \\
4 \\
\mathrm{I} 3 \\
\\
3 \\
4 \\
4 \\
6\end{array}$ & $\begin{array}{l}2-I-I-I \\
3-2-I-I \\
5-4-3-2-2-2-2-2-2 \\
\quad I-I-I-I \\
3-2-I \\
I 6-4-3-3 \\
\text { I } 1-9-9-9-8-7\end{array}$ & $\begin{array}{l}\mathrm{I}, 2 \\
\mathrm{I}, 7 \\
2, \mathrm{I} \\
\\
2 \\
6,5 \\
8,8\end{array}$ & $\begin{array}{l}I-2 \\
I-3 \\
I-5 \\
I-3 \\
3-I 6 \\
7-I I\end{array}$ \\
\hline
\end{tabular}

La dispersion des points (graphiques $I, 2,3$ ) indique une grande amplitude dans la réponse des ovaires, évaluée d'après le nombre de corps jaunes, pour une même dose d'hormone.

Les produits utilisés étant riches surtout en hormone de différenciation folliculaire ou F. S. H. (HAmmoND, I95I) des différences hypophysaires individuelles de teneur en $\mathrm{LH}$ pouvaient expliquer la variabilité des réponses, un manque d'hormone lutéinisante constituant un facteur limitant, comme permettait de le penser la présence d'un nombre parfois élevé de follicules non éclatés.

Dans ces conditions nous avons soumis des brebs recevant au $I_{3}$ jour du cycle par voie sous-cutanée 240 U. R. d'extrait hypophysaire, à des injections intraveineuses d'extrait hypophysaire de cheval (4O U. R.) et d'hormone chorionique ( 3 ooo U. I.) plus riche en LH.

TABLEAU II

\begin{tabular}{|c|c|c|c|c|c|}
\hline $\begin{array}{c}\text { Dose injectée } \\
\text { en sous-cutanée } \\
\text { (extrait hypo- } \\
\text { physaire de } \\
\text { cheval) }\end{array}$ & $\begin{array}{c}\text { Dose injectée } \\
\text { en } \\
\text { intra-veineuse }\end{array}$ & $\begin{array}{l}\text { Nombre de } \\
\text { brebis traitêes }\end{array}$ & $\begin{array}{c}\text { Nombre } \\
\text { d'ovulations }\end{array}$ & $\begin{array}{l}\text { Taux moyen } \\
\text { d'ovulation }\end{array}$ & $\begin{array}{c}\text { Variations } \\
\text { du taux } \\
\text { d'ovulation }\end{array}$ \\
\hline $\begin{array}{l}240 \text { U. R. } \\
24 \circ \text { U. R. } \\
24 \circ \text { U. R. }\end{array}$ & $\begin{array}{r}40 \text { U. R. } \\
240 \text { U. R. } \\
40 \text { U. R. } \\
+3000 \text { U.I. } \\
\text { chorionique }\end{array}$ & $\begin{array}{l}4 \\
4 \\
3\end{array}$ & $\left|\begin{array}{l}30-21-17-7 \\
23-17-14-9 \\
16-12-9\end{array}\right|$ & $\begin{array}{l}18,7 \\
16 \\
12,3\end{array}$ & $\begin{array}{l}7-30 \\
9-27 \\
9-16\end{array}$ \\
\hline
\end{tabular}

Du tableau II, il ressort qu'administré par voie intraveineuse en 

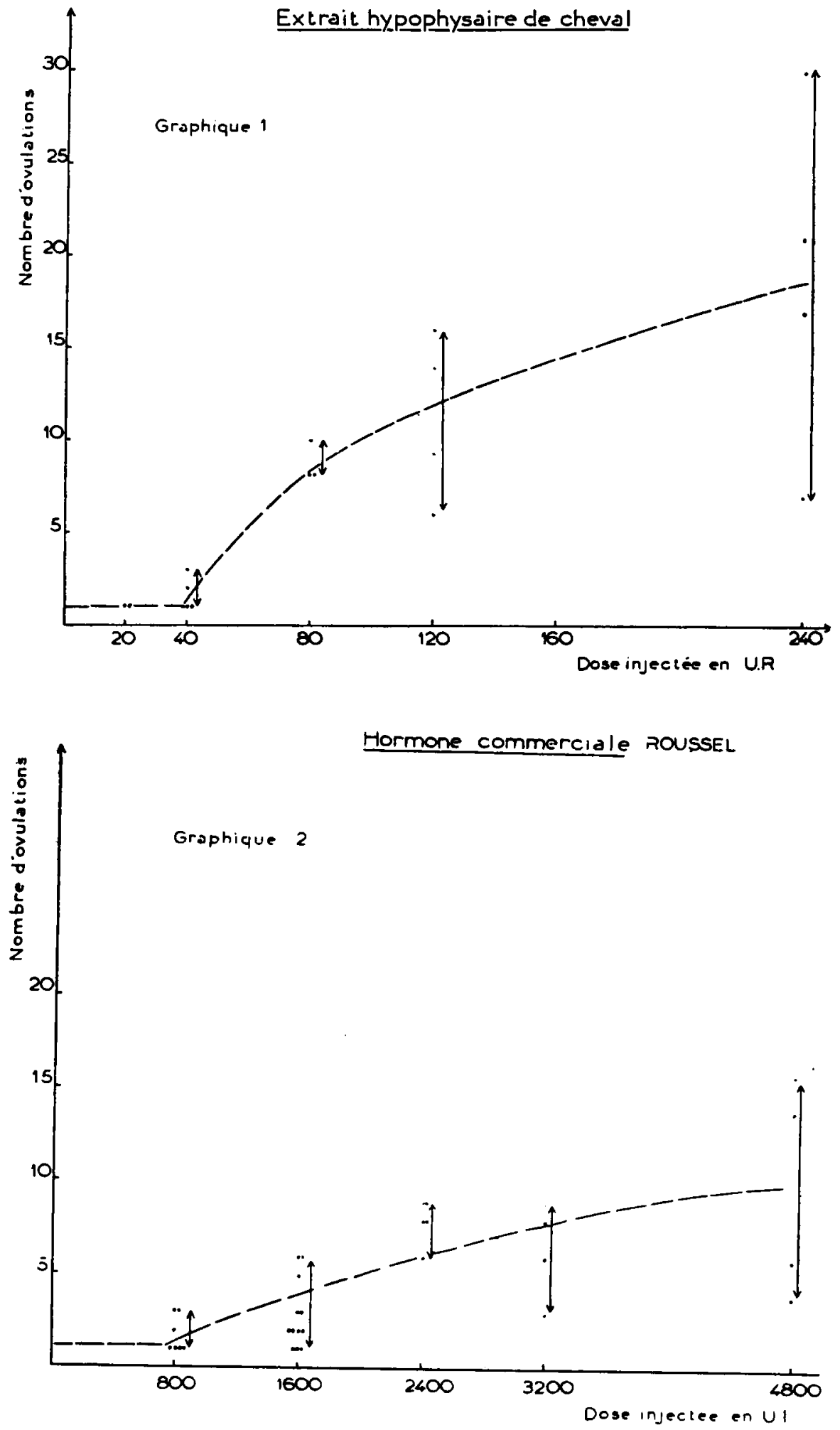
début d'œstrus, un supplément de LH n'améliore pas le taux d'ovulation. RoBINSON (I95I) avait d'ailleurs montré que l'hypophyse de brebis secrétait une quantité de $\mathrm{L}_{\mathrm{H}} \mathrm{H}$ permettant la ponte d'un grand nombre d'ovocytes. Le nombre de follicules différenciés non éclatés, est toutefois nettement plus élevé chez les animaux soumis à une injection intraveineuse de 240 U. R. d'extrait hypophysaire (moyenne de I2), que chez les brebis traitées avec l'hormone chorionique (moyenne de 2,7). L'extrait hypophysaire de cheval, injecté en début de rut, déterminerait la différenciation tardive de follicules qui n'éclatent pas.

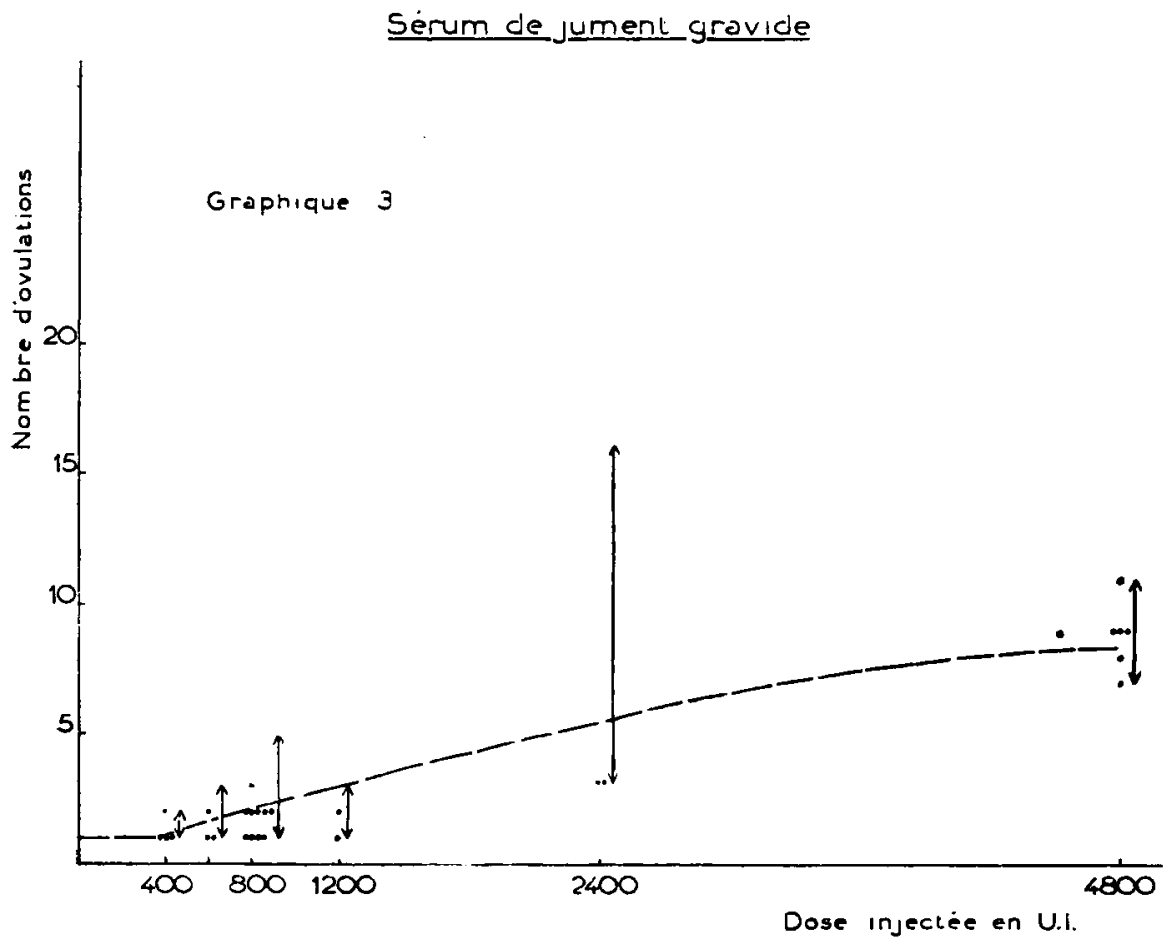

RoBinson a constaté une grande variabilité du taux d'ovulation, même avec des doses élevées de S. J. G. et d'autres auteurs en utilisant le même produit (Hammond, Hammond Jr. et Parkes, I942, ZavaDOwSKY, I945) étaient également arrivés à des résultats inconstants. Au contraire pour des doses élevées et quelle que soit l'origine de l'hormone, nous sommes parvenus à obtenir une ponte, régulière, constante et importante : 8 à Io ovocytes avec l'hormone hypophysaire, 6 à 9 avec l'hormone sérique, 7 à II avec le sérum de jument gravide; les moyennes des taux d'ovulation obtenus sont d'ailleurs très voisines et respectivement de $8,7,8$ et 8,8 . Ces valeurs sont obtenues avec des doses de $80 \mathrm{U}$. R. d'extrait hypophysaire contre 2400 U. I. d'hormone commerciale et 4800 U. I. de sérum de jument gravide. 
Des expériences non rapportées ici nous ont montré que cette homogénéité des réponses disparaissait aux approches de la période de repos sexuel.

Mais alors que Thibault, ORTAVANT et Laplaud (I948) semblaient avoir obtenu une méthode sûre et pratique de superovulation, nous enregistrons, avec des doses faibles, les seules intéressantes pour l'élevage, la même variabilité que RoBInson, aucune superovulation n'étant toutefois obtenue avec 400 U. I., contrairement à ce qu'affirme cet auteur.

Cette inconstance dans les résultats, en employant des techniques analogues, s'explique difficilement, RoBINson (I95I) a étudié l'influence de différents facteurs susceptibles d'affecter la réponse des ovaires aux hormones gonadotropes. Alors que l'auteur injectait $1^{\prime}$ hormone au $\mathrm{I}^{\mathrm{e}}$ et parfois au $\mathrm{I}^{\mathrm{e}}$ jour du cycle cestrien, nous n'avons pas obtenu des résultats. plus homogènes, en faisant l'injection de doses faibles d'hormone au

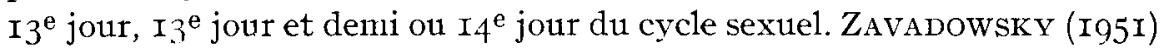
a cependant signalé que l'apport de vitamine $\mathrm{A}$ et $\mathrm{E}$ avant le traitement par le S J. G. pouvait modifier la réponse de l'animal.

En dehors de cette grande variabilité des réponses de part et d'autre de la dose optimum, il semble qu'une part importante des résultats. différents publiés, pour une dose apparemment identique, par différents auteurs, tienne à la méthode de dosage de l'hormone utilisée.

En effet le dosage est basé sur l'augmentation de poids des ovaires de rates impubères (COLE), alors que 1'hormone est utilisée pour provoquer un nombre plus ou moins élevé d'ovulations. Il conviendrait mieux que le dosage soit réalisé sur l'espèce que l'on étudie, compte tenu du but cherché. Aussi pour la brebis, il serait rationnel de considérer comme une unité brebis, la quantité d'hormone permettant d'obtenir une moyenne de 8 à 9 ovulations.

On peut donc affirmer que si l'on peut dans un but expérimental, obtenir une réponse régulière et importante des ovaires de brebis, il n'existe pas dans les circonstances actuelles, une méthode sûre de superovulation compatible avec les exigences de l'élevage.

\section{BIBLIOGRAPHIE}

COLE (H. H.). - On the biological properties of Mare gonadotrophic hormone. Amer. J. Anat., 2, 299-33I, I936.

HALEY (T. J.). - The preparation and properties of equine pituitary gonadotropin., J. Amer. Pharm. Ass., 39, I36-I37, I950.

Hammond (J.). - The practical use of hormones in animal production., Congrès de Zootechnie, Gand, I95I.

Hammond (J.), Hammond (J. Jr.), Parkes (A. S.). - Hormonal augmentation of fertility in sheep. I Introduction of ovulation, superovulation and heat in sheep., J. Agric. Sci., 32, 308-323, I942. 
(II, I953) VARIATIONS DES RÉPONSES DE I'OVAIRE DE LA BREBIS I95

Ortavant (R.), 'Thibaulit (C.), Wintenberger (S.). - Contribution à l'étude de la superovulation expérimentale chez la brebis. Ann. Endocr., 2, I70-I73, I949.

ThibaulT (C.), ORTAvant (R.), LAPlaud (M.). - Recherches sur la superovulation expérimentale chez la brebis, Ann. Endocr., 1, 83-89, I948.

Robinson (T. J.). - The control of fertility in sheep. Part. II The augmentation of fertility by gonadotrophin treatment of the ewe in the normal breeding season., $J$. Agric. Sci., 41, 6-63, I95I.

Zavadowski (B. M.). - Méthode hormonale des agnelages., Ogiz. Selchozgiz., I945.

ZaVAdosKI (M. M.). - The role of vitamin A et $\mathrm{E}$ in multifotation in sheep and the standardisation., of P. M. S., Dokl. Akad. seljskohoz. Nauk. Lenin., 16, 35-9, I95I. (A.B.A. 20, I55).

Le Directeur-Gérant : B. LACLAVIÈRE. 